\title{
Automorphisms and Non-integrability
}

\author{
JORGE V. PEREIRA and PERCY F. SÁNCHEZ
}

Instituto de Matemática Pura e Aplicada, Estrada D. Castorina, 110, 22460-320 Rio de Janeiro, RJ, Brasil

Manuscript received on November 19, 2004; accepted for publication on March 25, 2005; presented by MANFREDO DO CARMO

\begin{abstract}
On this note we prove that a holomorphic foliation of the projective plane with rich, but finite, automorphism group does not have invariant algebraic curves.
\end{abstract}

Key words: Holomorphic Foliations, Jouanolou Theorem.

\section{INTRODUCTION}

On the monograph (Jouanolou 1979) it is proved that for every $d \geq 2$, there exists a residual subset of the space of degree $d$ holomorphic foliations of the projective plane whose elements do not have any invariant algebraic curve. The hard part of the proof is to exhibit an explicit example having such property. Jouanolou shows that the foliations induced by the homogeneous 1 -forms

$$
\Omega_{d}=\operatorname{det}\left(\begin{array}{ccc}
x & y & z \\
y^{d} & z^{d} & x^{d} \\
d x & d y & d z
\end{array}\right),
$$

where $d \geq 2$, have such properties. His proof explores the richness of the automorphism groups of such examples.

In (Zoladek 1998) new examples of foliations of the projective plane without invariant algebraic curves are constructed. These examples also have rich automorphism groups.

In (Maciejewski et al. 2000), the four authors prove that some higher dimensional analogues of Jouanolou's examples do not have invariant algebraic hypersurfaces. Again the automorphism group of these foliations play a major role on the course of the proof.

Besides taking advantage of a rich automorphism group the above mentioned works make use of the explicit form of the equations investigated and impose some restrictions on the singularities of the equations.

Correspondence to: Jorge Vitório Pereira

E-mail: jvp@impa.br 
The purpose of this note is to settle a general result for foliations of the projective plane with rich automorphism group. Unfortunately we cannot completely get ride of the hypothesis on the singular set of the foliation, although our assumptions are on the eigenvalues of just one of the singularities. More precisely we prove the following

THEOREM 1. Let $\mathcal{F}$ be a holomorphic foliations foliations of $\mathbb{P}^{2}$. Suppose that $\mathcal{F}$ has at least one singularity with non-singular linear part and whose quotient of eigenvalues is not rational nor a root of a unity. If the automorphism group of $\mathcal{F}$ is finite and acts without nontrivial fixed points on the space of cofactors then $\mathcal{F}$ does not admit any invariant algebraic curve.

For a definition of the space of cofactors and the action of automorphism group of the foliation on it the reader should consult section 2 .

\section{COFACTOR REPRESENTATION}

On this paper a degree $d$ singular holomorphic foliation of $\mathbb{P}^{2}$ will be given by a 1 -form $\omega$ on $\mathbb{C}^{3}$ annihilated by the radial vector field with homogeneous coefficients of degree $d+1$. More explicitly if $\omega=A d x+B d y+C d z$ then $A, B$ and $C$ are homogenous polynomials of degree $d+1$ and $x A+y B+z C=0$. We will denote the foliation induced by $\omega$ by $\mathcal{F}_{\omega}$.

The automorphism group of $\omega$ is the subgroup of $S L(3, \mathbb{C})$ defined by

$$
\operatorname{Aut}(\omega)=\left\{\phi \in \operatorname{SL}(3, \mathbb{C}) \mid \phi^{*} \omega \wedge \omega=0\right\} .
$$

The automorphism group of $\mathcal{F}_{\omega}$ is the image of $\operatorname{Aut}(\omega)$ by the natural projection $\operatorname{SL}(3, \mathbb{C}) \rightarrow$ $\operatorname{PSL}(3, \mathbb{C})$, i.e., $\operatorname{Aut}\left(\mathcal{F}_{\omega}\right)=\operatorname{P}(\operatorname{Aut}(\omega))$.

Note that if $\phi \in \operatorname{Aut}(\omega)$ then there exists a non zero complex number $\lambda(\phi)$ such that $\phi^{*} \omega=$ $\lambda(\phi) \cdot \omega$. Moreover,

$$
\begin{aligned}
\lambda: \operatorname{Aut}(\omega) & \rightarrow \mathbb{C}^{*} \\
\phi & \rightarrow \lambda(\phi)
\end{aligned}
$$

is a homomorphism of groups.

If $F \in \mathbb{C}[x, y, z]$ is a homogeneous polynomial describing an invariant algebraic curve for $\mathcal{F}$ then

$$
d F \wedge \omega=F \Theta_{F}
$$

where $\Theta_{F}$ is a 3-form satisfying the following

Lemma 1. There exists a unique 3-form $\beta_{F}$ such that

$$
\Theta_{F}=\frac{\operatorname{deg}(F)}{d+2} \cdot d w+i_{R} \beta_{F} .
$$

Proof. Applying $i_{R}$ to both sides of $d F \wedge \omega=F \Theta_{F}$ we have

$$
i_{R} \Theta_{F}=\operatorname{deg}(F) \cdot \omega .
$$


It follows from Euler's formula that $i_{R}(d \omega)=(d+2) \omega$ and consequently

$$
i_{R}\left(\Theta_{F}-\frac{\operatorname{deg}(F)}{d+2} \cdot d \omega\right)=0 \text {. }
$$

The existence and uniqueness of $\beta_{F}$ follow from the De Rham's division Theorem.

From the above Lemma it follows that for every automorphism $\phi$ of $\omega$ the following identity holds

$$
\beta_{\phi^{*} F}=\frac{\phi^{*} \beta_{F}}{\lambda(\phi)}
$$

If $\mathcal{F}$ is a foliation of degree $d$ then $\beta_{F}$ has degree $d-1$. Denote by $\Omega_{d-1}^{3}$ the space of homogeneous 3-forms on $\mathbb{C}^{3}$ of degree $d-1$. Hence we have a representation $\Phi_{\omega}: \operatorname{Aut}(\omega) \rightarrow$ $\operatorname{Hom}\left(\Omega_{d-1}^{3}, \Omega_{d-1}^{3}\right)$ defined by

$$
\Phi_{\omega}(\phi)=\left(\beta \mapsto \frac{\phi^{*} \beta}{\lambda(\phi)}\right) .
$$

Since $\Phi_{\omega}(\mu \cdot \mathrm{Id})=\mathrm{Id}$ for every $\mu \in \mathbb{C}^{*}$, it follows that $\Phi_{\omega}=\Phi_{\mathcal{F}_{\omega}} \circ \pi$ where $\pi: \operatorname{Aut}(\omega) \rightarrow$ $\operatorname{Aut}\left(\mathcal{F}_{\omega}\right)$ is the natural projection and

$$
\Phi_{\mathcal{F}_{\omega}}: \operatorname{Aut}\left(\mathcal{F}_{\omega}\right) \rightarrow \operatorname{Hom}\left(\Omega_{d-1}^{3}, \Omega_{d-1}^{3}\right)
$$

is, by definition, the cofactor representation. We will say that the automorphism group of $\mathcal{F}$ acts without nontrivial fixed points on the space of cofactors if

$$
\Phi_{\mathcal{F}_{\omega}}(\phi)(\beta)=\beta, \forall \phi \in \operatorname{Aut}(\mathcal{F}) \backslash \text { and for some } \beta \in \Omega_{d-1}^{3} \text { then } \beta=0 \text {. }
$$

Proposition 1. Let $\mathcal{F}$ be a foliation on $\mathbb{P}^{2}$. If the automorphism group of $\mathcal{F}$ is finite and acts without nontrivial fixed points on the space of cofactors then either $\mathcal{F}$ admits liouvillian first integral or $\mathcal{F}$ does not admit an invariant algebraic curve.

Proof. Suppose that $\mathcal{F}$ admits an invariant algebraic curve $C$ given by homogeneous polynomial $F$. If we consider the homogeneous polynomial

$$
G=\prod_{\sigma \in \operatorname{Aut}(\mathcal{F})} \sigma^{*} F
$$

we see that $G$ is invariant by both $\mathcal{F}$ and $\operatorname{Aut}(\mathcal{F})$. Consequently the cofactor associated to $G$ is a fixed point for $\Phi_{\mathcal{F}_{\omega}}$ and therefore, by hypothesis, is equal to zero. Consequently, it follows from Lemma 1 that

$$
d \omega=\frac{d+2}{\operatorname{deg}(G)} \cdot \frac{d G}{G} \wedge \omega .
$$

To conclude one has to observe that the multivalued 1-form $G^{-\frac{d+2}{\operatorname{deg}(G)}} \cdot \omega$ is closed and induces a closed multivalued one-form on the projective plane. In particular $\mathcal{F}$ admits a liouvillian first integral. 
EXAMPLE 1. Let $H$ be the subgroup of $\operatorname{Aut}\left(\mathbb{P}^{2}\right)$ generated by $\phi_{1}[x: y: z]=[-x:-y: z]$ and $\phi_{2}[x: y: z]=[-x: y:-z]$. Among the degree 2 foliations invariant by $G$ there exists a $\mathbb{P}^{2} \subset \operatorname{Fol}(2)=\mathbb{P H}^{0}\left(\mathbb{P}^{2}, \Omega^{1}(4)\right)$ whose elements are induced by the 1 -forms

$$
\omega_{(a, b, c)}=\left(a x y^{2}+b x z^{2}\right) d x+\left(-a x^{2} y+c y z^{2}\right) d y+\left(-b x^{2} z-c y^{2} z\right) d z .
$$

The cofactor representation is given by

$$
\begin{aligned}
& \Phi\left(\phi_{1}\right)((\alpha x+\beta y+\gamma z) d x \wedge d y \wedge d z)=(-\alpha x-\beta y+\gamma z) d x \wedge d y \wedge d z, \\
& \Phi\left(\phi_{2}\right)((\alpha x+\beta y+\gamma z) d x \wedge d y \wedge d z)=(-\alpha x+\beta y-\gamma z) d x \wedge d y \wedge d z .
\end{aligned}
$$

In particular $H$ acts without nontrivial fixed points. The lines $\left\{a y^{2}+b z^{2}=0\right\}$ and $\left\{-a x^{2}-c z^{2}=0\right\}$ are invariant by both $\mathcal{F}_{(a, b, c)}$ and $\operatorname{Aut}\left(\mathcal{F}_{a, b, c}\right)$. Consequently

$$
\frac{d\left(\left(a y^{2}+b z^{2}\right) \cdot\left(-a x^{2}-c z^{2}\right)\right)}{\left(a y^{2}+b z^{2}\right) \cdot\left(-a x^{2}-c z^{2}\right)} \wedge \omega=d \omega,
$$

and $\mathcal{F}_{(a, b, c)}$ has a liouvillian first integral as predicted by Proposition 1 . In fact one can easily verify that $\mathcal{F}_{(a, b, c)}$ has a rational first integral.

\section{PROOF OF THE MAIN RESULT}

Before starting the proof of the main result let us state a simple lemma

Lemma 2. Let $X$ be a holomorphic vector field on a neighborhood of $0 \in \mathbb{C}^{2}$ and $\omega=i_{X} d x \wedge d y$ a 1-form dual to $X$. Assume that 0 is a singularity of $X$. If there exists a 1-form $\eta$ such that $d \omega=\eta \wedge \omega$ then the trace of $D X(0)$ is zero.

Proof. Since $\omega=i_{X} d x \wedge d y$ we have $d \omega=d i v(X) d x \wedge d y=(\operatorname{Tr}(D X(0))+$ h.t.o $) d x \wedge d y$. Now as 0 is a singularity of $X$ we have:

$$
(\eta \wedge \omega)(0)=0=\operatorname{Tr}(D X(0)) d x \wedge d y
$$

Let $p$ be the reduced singularity of $\mathcal{F}$ whose quotient of eigenvalues is not rational nor a root of unity. If $\mathcal{F}$ admits an invariant algebraic curve $C$ we can suppose without loss of generality that $C$ is reduced and invariant by $\operatorname{Aut}(\mathcal{F})$. Moreover we can also assume that $\operatorname{Aut}(\mathcal{F})$ acts transitively on the set of irreducible components of $C$.

It follows from Lemma 1 that

$$
d \omega=\frac{d+2}{\operatorname{deg}(F)} \cdot \frac{d F}{F} \wedge \omega,
$$

where $F$ is a reduced homogeneous polynomial defining $C$.

From Lemma 2 we have $p \in C$. Since $p$ is reduced, we can choose local coordinates $(x, y)$ where $p=(0,0)$ and $\mathcal{F}$ is defined in these coordinates by

$$
\omega=\lambda x d y-y d x+\text { h.o.t. }
$$


Suppose that $C$ is smooth at $p$ and locally defined by either $\{x=0\}$ or $\{y=0\}$. From (1) and (2) we obtain in both cases that $\lambda$ is rational contradicting our hypothesis. Therefore $C$ will have two branches passing through $p$. In this case

$$
\frac{d(x y)}{x y} \wedge \omega=(\lambda+1) d x \wedge d y+\text { h.o.t. }=d \omega+\text { h.o.t. }
$$

Comparing this last equality with (1) we deduce that $d+2=\operatorname{deg}(F)$ and that the foliation is given by the closed 1-form $\frac{\omega}{F}$ with simple poles along $C$. By Theorem 2.1 of (Cerveau and Mattei 1982) it follows that $\mathcal{F}$ is defined by a closed meromorphic 1 -form

$$
\sum_{i=1}^{k} \alpha_{i} \frac{d F_{i}}{F_{i}}
$$

where $F_{i}$ are the irreducible factors of $F, F=F_{1} F_{2} \cdots F_{k}$. Writing the closed 1-form above in local coordinates $(x, y)$ this implies that $x$ and $y$ divide locally two distinct polynomials $F_{i}$, since otherwise the quotient of eigenvalues at $p$ would be 1 .

Without loss of generality we may suppose that $x$ divides $F_{1}$ and $y$ divides $F_{2}$. Thus we obtain that

$$
0=\left(\sum_{i=1}^{k} \alpha_{i} \cdot \frac{d F_{i}}{F_{i}}\right) \wedge \omega=\left(\lambda \alpha_{1}+\alpha_{2}\right) d x \wedge d y+\text { h.o.t. }
$$

Consequently we have $\lambda=-\frac{\alpha_{2}}{\alpha_{1}}$.

We can suppose without loss of generality that there exists $\phi \in \operatorname{Aut}(\mathcal{F})$ such that $\phi^{*} \frac{d F_{1}}{F_{1}}=\frac{d F_{2}}{F_{2}}$. Applying $\phi$ to $\frac{\omega}{F}$ we see that there exists a root of unity $\beta$ such that

$$
\phi^{*}\left(\sum_{i=1}^{k} \alpha_{i} \cdot \frac{d F_{i}}{F_{i}}\right)=\beta \cdot\left(\sum_{i=1}^{k} \alpha_{i} \cdot \frac{d F_{i}}{F_{i}}\right)
$$

Comparing the coefficients of $\frac{d F_{2}}{F_{2}}$ in both sides of the equation we infer that $\alpha_{1}=\beta \alpha_{2}$. Therefore $\lambda=-\beta^{-1}$ contrary to our hypothesis.

\section{SOME EXAMPLES}

\section{I The EXAmples of Jouanolou}

As stated in the introduction the examples of Jouanolou are the foliations of $\mathbb{P}^{2}$ induced by the 1 -forms $\omega_{d}=i_{R} i_{X_{d}} d x \wedge d y \wedge d z$, where $d \geq 2$ and $X_{d}=y^{d} \frac{\partial}{\partial x}+z^{d} \frac{\partial}{\partial y}+x^{d} \frac{\partial}{\partial z}$. We will denote the foliation induced by $\omega_{d}$ by $\mathcal{J}_{d}$. The automorphisms group of $\mathcal{J}_{d}$ is isomorphic to the semidirect product of $\mathbb{Z}_{3}$ and $\mathbb{Z}_{d^{2}+d+1}$. In particular it has order $3 \cdot\left(d^{2}+d+1\right)$. It is generated by $\phi_{1}[x: y: z]=[z: x: y]$ and $\phi_{2}[x: y: z]=\left[\delta x: \delta^{d} y: \delta^{d^{2}}\right]$ where $\delta$ is a $d^{2}+d+1$-root of the unity. 
The cofactor representation is given by

$$
\begin{aligned}
& \Phi_{d}\left(\phi_{1}\right)(F(x, y, z) d x \wedge d y \wedge d z)=F(z, x, y) d x \wedge d y \wedge d z \\
& \Phi_{d}\left(\phi_{2}\right)(F(x, y, z) d x \wedge d y \wedge d z)=F\left(\delta x, \delta^{d} y, \delta^{d^{2}} z\right) d x \wedge d y \wedge d z .
\end{aligned}
$$

Lemma 3. If $d \not \equiv 1$ mod 3 then the automorphism group of $\mathcal{J}_{d}$ acts without nontrivial fixed points on the space of cofactors.

PROOF. The eigenvalue of $\Phi_{d}\left(\phi_{2}\right): \Omega_{d-1}^{3} \rightarrow \Omega_{d-1}^{3}$ corresponding to the eigenvector $x^{i} y^{j} z^{k} d x \wedge$ $d y \wedge d z, i+j+k=d-1$, is given by $\delta^{i+j d+k d^{2}}$. To prove the lemma it is sufficient to show that for $d \not \equiv 1 \bmod 3$ the system

$$
\begin{aligned}
i+j+k & =d-1 \\
i+d j+d^{2} k & \equiv 0 \bmod \left(d^{2}+d+1\right),
\end{aligned}
$$

has no solutions with $i, j$ and $k$ nonnegative integers.

Subtracting the first equation from the second we obtain that

$$
(d-1)(j+(d+1) k) \equiv-(d-1) \bmod \left(d^{2}+d+1\right) .
$$

Since $d^{2}+d+1=(d+2)(d-1)+3$ we see that the greatest common divisor of $d^{2}+d+1$ and $d-1$ is 1 whenever $d \not \equiv 1 \bmod 3$. Therefore from our assumptions we have that

$$
j+(d+1) k \equiv-1 \quad \bmod \left(d^{2}+d+1\right),
$$

and $j, k \geq 0$ implies that $j+(d+1) k \geq d^{2}+d$.

Since $j, k \leq d-1$ we obtain that $j+(d+1) k \leq d^{2}+d-2$. This contradiction is sufficient to settle the lemma.

From the lemma above and Theorem 1 we obtain a proof that $\mathcal{J}_{d}$ has non algebraic solutions when $d \neq 1 \bmod 3$. Note that this result also holds for every integer $d$ greater than or equal to 2 , see (Jouanolou 1979).

\subsection{ONE OF ZOLADEK's EXAMPLES}

Denote by $\mathcal{F}_{[a: b: c]},[a: b: c] \in \mathbb{P}^{2}$, the degree 3 foliations of $\mathbb{P}^{2}$ defined by the 1 -forms

$$
\omega_{a, b, c}=\left(a x^{2} y^{2}+b x z^{3}\right) d x+\left(-a x^{3} y+c y^{2} z^{2}\right) d y+\left(-b x^{2} z^{2}-c y^{3} z\right) d z .
$$

These foliations are invariant by the automorphism

$$
A[x: y: z]=\left[\delta x: \delta^{2} y: \delta^{4} z\right]
$$

where, $\delta$ is the seventh root of unity.

A simple computation shows that $A$ acts without non-trivial fixed points in $\Omega_{2}^{3}$.

When $a \cdot b \cdot c \neq 0$, the singularities distinct from $\{[0: 0: 1],[0: 1: 0],[1: 0: 0]\}$ have as quotient of eigenvalues $\frac{11+5 \sqrt{3} i}{14}$. Therefore from Theorem 1 the foliation $\mathcal{F}_{[a: b: c]}$ has no algebraic solutions when $a \cdot b \cdot c \neq 0$. 


\section{ACKNOWLEDGMENTS}

Jorge Vitório Pereira is supported by Conselho Nacional de Desenvolvimento Científico e Tecnológico (PROFIX-CNPq).

\section{RESUMO}

Seja $\mathcal{F}$ uma folheação do plano projetivo complexo de grau $d$ com grupo de automorfismo finito e cuja ação no espaço de cofatores não possui ponto fixo. Neste artigo mostramos que se $\mathcal{F}$ possui ao menos uma singularidade genérica então $\mathcal{F}$ não possui nenhuma curva algébrica invariante.

Palavras-chave: Folheações Holomorfas, Teorema de Jouanolou.

\section{REFERENCES}

Cerveau D And Mattei J-F. 1982. Formes intégrables holomorphes singulières, Astérisque 97, SMF. JouAnolou J-P. 1979. Equations of Pfaff algébriques, Lect Notes in Math 708, Springer Verlag, Berlin.

Maciejewski A, Ollagnier J, Nowwicki A and Strelcyn J-M. 2000. Around Jouanolou non-integrability theorem. Indag Mathem 11: 239-254.

Zoladek H. 1998. New examples of holomorphic foliations without algebraic leaves. Stud Math 131: $137-142$. 